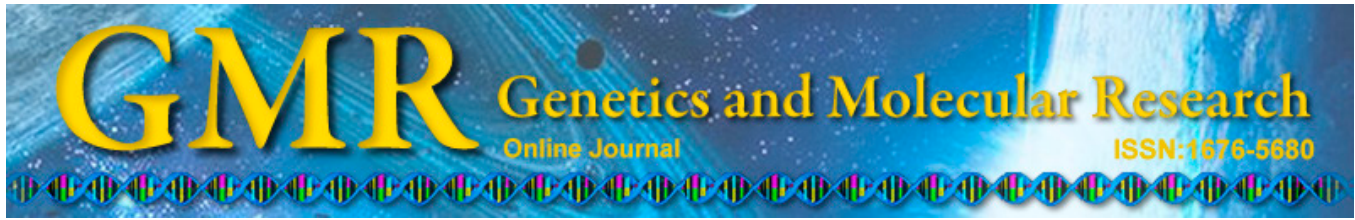

\title{
Analysis of the genetic diversity of Chinese native Cannabis sativa cultivars by using ISSR and chromosome markers
}

\author{
L.G. Zhang ${ }^{1,2}$, Y. Chang ${ }^{1}$, X.F. Zhang ${ }^{3}$, F.Z. Guan' ${ }^{2}$ H.M. Yuan ${ }^{2}$, Y. Yu ${ }^{2}$ \\ and L.J. Zhao ${ }^{4}$ \\ ${ }^{1}$ College of Life Science, Northeast Agricultural University, Harbin, China \\ ${ }^{2}$ Institute of Industrial Crops, Heilongjiang Academy of Agricultural Sciences, \\ Harbin, China \\ ${ }^{3}$ College of Mathematics and Information Sciences of Guangxi University, \\ Nanning, China \\ ${ }^{4}$ Institute of Crop Breeding, Heilongjiang Academy of Agricultural Sciences, \\ Harbin, China
}

Corresponding author: Y. Chang

E-mail: yingchangnew@163.com

Genet. Mol. Res. 13 (4): 10490-10500 (2014)

Received January 16, 2014

Accepted September 22, 2014

Published December 12, 2014

DOI http://dx.doi.org/10.4238/2014.December.12.10

\begin{abstract}
Hemp (Cannabis sativa) is an important fiber crop, and native cultivars exist widely throughout China. In the present study, we analyzed the genetic diversity of 27 important Chinese native hemp cultivars, by using inter-simple sequence repeats (ISSR) and chromosome markers. We determined the following chromosome formulas: $2 n=20=14 \mathrm{~m}+6 \mathrm{sm} ; 2 n=20=20 \mathrm{~m} ; 2 n=20=18 \mathrm{~m}+$ $2 \mathrm{sm} ; 2 n=20=16 \mathrm{~m}+4 \mathrm{sm}$; and $2 n=20=12 \mathrm{~m}+8 \mathrm{sm}$. The results of our ISSR analysis revealed the genetic relationships among the 27 cultivars; these relationships were analyzed by using the unweighted pair-group method based on DNA polymorphism. Our results revealed that all of the native cultivars showed considerable genetic diversity. At a genetic distance of 0.324 , the 27 varieties could be classified into
\end{abstract}


five categories; this grouping corresponded well with the chromosome formulas. All of the investigated hemp cultivars represent relatively primitive types; moreover, the genetic distances show a geographical distribution, with a small amount of regional hybridity.

Key words: Cannabis sativa; Inter-simple sequence repeat; Genetic diversity; Chromosome

\section{INTRODUCTION}

Cannabis has been cultivated as a crop since ancient times, because of its industrial, agricultural, and medicinal value, especially in the textile and paper industries. Cannabis has unique properties, and therefore cannot be replaced by other crops. However, the gradual introduction, exchange, and wide distribution of cannabis germplasm have resulted in confusion regarding the naming of cultivars. In addition, a single cultivar may show variations when planted in different regions over a long period of time. The range of cannabis genetic variation is relatively large, and the genome variation is $>1 \%$, which is 10 times higher than that of the human genome (van Bakel et al., 2011). Cannabis originated in China, and some native cultivars have been developed by planting for thousands of years, thereby producing homogeneity under different names or heterogeneity under the same name. Consequently, the field performance of a single germplasm may vary markedly, and this has resulted in a lack of criteria for seed production and variety protection. Typically, higher fiber content is obtained when southern varieties are planted in northern China; however, these varieties do not seed because cannabis is a short-day plant. During the growing season, the day length in northern China is longer than in southern China, and this increases the vegetative growth period. Confusion regarding management practices has affected seed production, and there has been a considerable reduction in production in northern China, caused by the planting of mislabeled or fake cannabis seeds; in addition, germplasm has been repeatedly exchanged during the breeding process, thereby wasting valuable time and resources. On the other hand, cannabis germplasm is frequently dioecious and is therefore easily cross-fertilized. Thus, it is very important to establish methods and standards for distinguishing genetic variation among cannabis germplasm.

Several previous studies have examined cannabis genetic diversity; however, these investigations have focused on traditional morphological classification (Hong and Clarke, 1996; Wibowo et al., 2004). Morphological traits are easily affected by certain factors, including the environment, physiology, and growth stage; moreover, some traits are not exhibited until maturity. Therefore, morphological markers have limited validity for the study of genetic variation. Faeti et al. (1996) investigated the genetic diversity of 13 germplasms by using 10 RAPD (random amplified polymorphic DNA) primers, and proposed that RAPD markers represent a powerful tool for the investigation of genetic variation in cannabis cultivars.

Chromosomal variation is a cytological marker that has unique advantages for studying the origin and evolution of species. These advantages include species-specific chromosome information, very high stability, and reproducibility. The number of chromosomes and karyotype data within a species are fairly constant; however, even within species having the same number of chromosomes, the chromosome types and locations may differ. To date, few cytological studies on cannabis germplasm have been conducted; however, such studies can 
provide useful information when elucidating genetic relationships, evaluating germplasm, and examining species origins and evolution based on chromosome traits (Babiker et al., 1991; Passamonti et al., 1999).

In comparison with many other marker systems such as AFLP, SSR, RAPD, and RFLP, ISSR markers (Zietkiewicz et al., 1994) are simple to use, and show improved stability and polymorphism (Nagaoka and Ogihara, 1997; Qian et al., 2001; Wu et al., 2004); in addition, they do not require previous genomic sequence information for polymorphism analysis (Bornet and Branchard, 2001). Consequently, ISSR markers represent a powerful tool for investigating genetic variation within a species (Culley and Wolfe, 2001; Vicente et al., 2011; Rubio-Moraga et al., 2012). ISSR markers have been widely used for investigating population genetics in various plant species (Gajera et al., 2010; Wang et al., 2012; Zhang et al., 2013; Chen et al., 2013; Lin and Hu, 2013). In the present study, we used ISSR markers to investigate genetic diversity in cannabis.

Chromosome markers demonstrate the results of accumulated changes and different stages, whereas ISSR markers enable the quantification of genetic variation. Thus, in the present study, we used chromosome markers and ISSR markers to obtain objective and comprehensive results.

\section{MATERIAL AND METHODS}

\section{Experimental materials}

The 27 important native cannabis cultivars, which had been planted in different areas of China since the 1950s, were collected by the Institute of Industrial Crops in December 2010, Heilongjiang Academy of Agricultural Sciences, Harbin, China.

We used a Takara Genomic DNA Extraction Kit (Takara, Dalian, China) to isolate genomic DNA from each sample. The quality of each DNA sample was detected by using $1 \%$ agarose gel electrophoresis. We measured the concentration and purity of each sample by using ultraviolet light.

For ISSR analysis, 100 primers were synthesized by Shanghai Sangon Biological Engineering Co. Ltd. (Sangon, Shanghai, China), using sequences released by the University of British Columbia.

\section{Chromosome analysis}

We used the wall degradation hypotonic method to prepare chromosome samples from each plant. Seeds were cultured in distilled water for $24 \mathrm{~h}$ at $25^{\circ} \mathrm{C}$, until the roots were approximately $1 \mathrm{~cm}$ long. The root tips were excised and placed in $1.5 \%$ santochlor solution at $10^{\circ} \mathrm{C}$ for $5 \mathrm{~h}$, followed by Carnoy's solution for $12 \mathrm{~h}$; finally, the roots were placed in $70 \%$ alcohol. After 4\% cellulase degradation of the cell walls, the samples were stained with $10 \%$ Giemsa for chromosome sectioning. The samples were examined under a Leica DM4000 microscope.

\section{ISSR analysis}

We used a gradient factor experimental design to optimize the influences of 5 factors, 
namely, dNTPs, Taq DNA polymerase, primer concentrations, pre-degeneration time, and annealing temperature, on PCR amplification. In this way, we established a suitable ISSR PCR system for hemp. Each $25-\mu \mathrm{L}$ reaction volume included $200 \mu \mathrm{M}$ dNTPs, $1 \mathrm{ng} / \mu \mathrm{L}$ template, $200 \mathrm{nM}$ primers, and $1 \mathrm{U}(2.5 \mathrm{pmol} / \mu \mathrm{L})$ Taq. The amplification program used was as follows: $94^{\circ} \mathrm{C}$ for $4 \mathrm{~min}$; followed by 35 cycles each of denaturation at $94^{\circ} \mathrm{C}$ for $20 \mathrm{~s}$, annealing at $48^{\circ} \mathrm{C}$ for $50 \mathrm{~s}$, and extension at $48^{\circ} \mathrm{C}$ for $1 \mathrm{~min}$; and a final cycle at $72^{\circ} \mathrm{C}$ for $10 \mathrm{~min}$. The products were stored at $4^{\circ} \mathrm{C}$. PCR was performed by using an $\mathrm{S} 1000^{\mathrm{TM}}$ Thermal Cycler (Bio-Rad, USA). We screened 23 of the initial 100 primers by using our stable ISSR reaction system (Table 1). Electrophoresis bands that repeatedly appeared in 2 experiments were used for data analysis.

\begin{tabular}{llcl}
\multicolumn{2}{l}{ Table 1. Screened primers for cannabis ISSR analysis. } \\
\hline Serial number & Primer sequence (5'-3') & Serial number & Primer sequence (5'-3') \\
\hline 804 & TAT ATA TAT ATA TAT AA & 852 & TCT CTC TCT CTC TCT CRA \\
808 & AGA GAG AGA GAG AGA GC & 857 & ACA CAC ACA CAC ACA CYG \\
812 & GAG AGA GAG AGA GAG AA & 859 & TGT GTG TGT GTG TGT GRC \\
817 & CAC ACA CAC ACA CAC AA & 867 & GGC GGC GGC GGC GGC GGC \\
819 & GTG TGT GTG TGT GTG TA & 869 & GTT GTT GTT GTT GTT GTT \\
824 & TCT CTC TCT CTC TCT CG & 872 & GAT AGA TAG ATA GAT A \\
827 & ACA CAC ACA CAC ACA CG & 873 & GAC AGA CAG ACA GACA \\
834 & AGA GAG AGA GAG AGA GYT & 880 & GGA GAG GAG AGG AGA \\
837 & TAT ATA TAT ATA TAT ART & 885 & BHB GAG AGA GAG AGA GA \\
840 & GAG AGA GAG AGA GAG AYT & 889 & DBD ACA CAC ACA CAC A \\
841 & GAG AGA GAG AGA GAG AYC & 892 & TAG ATC TGA TAT CTG AAT TCC C \\
848 & CAC ACA CAC ACA CAC ARG & & \\
\hline
\end{tabular}

\section{Statistical analysis}

Chromosome types were classified following Lin and Chen (1983). The karyotypes were classified according to Stebbins (1971). The relative length of each chromosome was calculated by using the Kuo method. The asymmetrical karyotype coefficient was calculated by using the Arano method. The electrophoresis results were translated into a binary data matrix according to the presence (1) or absence (0) of bands (Williams et al., 1990). Estimations of genetic distance and genetic similarity were performed according to Nei and Li (1979).

\section{RESULTS}

\section{Chromosome analysis}

We identified the following 5 chromosome types among the 27 native cannabis cultivars: $2 n=20=14 \mathrm{~m}+6 \mathrm{sm} ; 2 n=20=20 \mathrm{~m} ; 2 n=20=18 \mathrm{~m}+2 \mathrm{sm} ; 2 n=20=16 \mathrm{~m}+4 \mathrm{sm}$; and $2 n=20=12 \mathrm{~m}+8 \mathrm{sm}$. In addition, we identified 3 karyotypes, namely, 1B, 2B, and 2A (Figure 1). For all samples, the chromosome number was 20 , and this mainly comprised metacentric and submetacentric chromosomes. However, the constitution of chromosomes differed in terms of the asymmetry coefficient, which ranged from 54.52 to $61.12 \%$, and also in terms of the length ratios (Table 2). The 27 native cannabis cultivars were distributed throughout China (Table 2 and Figure 2). 

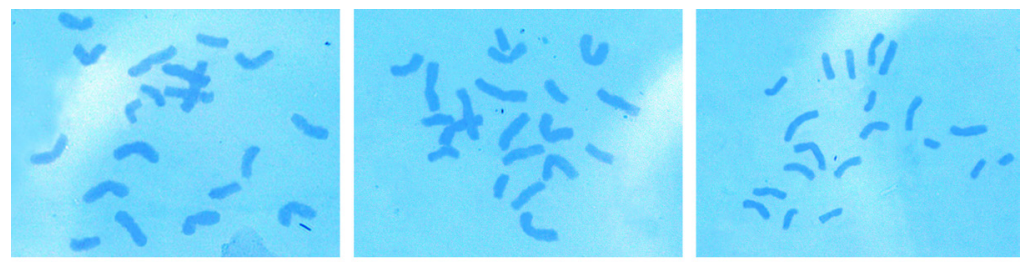

(a)

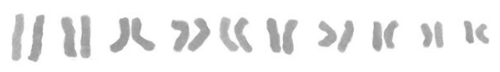

Nutexisun

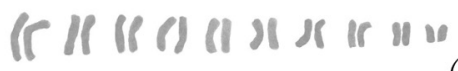

Hillititii

(b)

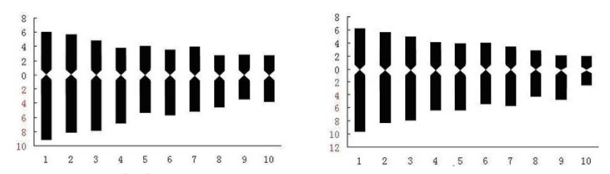

(c)

Figure 1. Analysis of genetic diversity by evaluation of chromosome data. a. Chromosome sectioning: left, 'Tongxiang' cultivar; middle, 'Xiangyun' cultivar; right, 'Wuchang' cultivar. b. Chromosome pairing: upper, 'Tongxiang'; middle, 'Xiangyun'; lower, 'Wuchang'. c. Idiograms of karyotype: left, 1B ('Tongxiang'); middle, 2A ('Xiangyun'); right, 2B ('Wuchang').

Table 2. Chromosome differences among the 27 native cannabis cultivars.

\begin{tabular}{|c|c|c|c|c|c|c|}
\hline Serial number & Name of germplasm & $\begin{array}{r}\text { Asymmetry } \\
\text { coefficient }\end{array}$ & $\begin{array}{c}\text { Length ratio } \\
\text { of chromosomes }\end{array}$ & Karyotype & Karyotypic formula & Distribution (Provinces) \\
\hline 1 & Wuchang & $61.12 \%$ & 3.45 & $2 \mathrm{~B}$ & $2 n=20=18 \mathrm{~m}+2 \mathrm{sm}$ & Heilongjiang, Jilin \\
\hline 2 & Boli & $55.12 \%$ & 2.12 & $1 \mathrm{~B}$ & $2 n=20=14 m+6 s m$ & Heilongjiang \\
\hline 3 & Yongning & $55.44 \%$ & 2.31 & $1 \mathrm{~B}$ & $2 n=20=14 m+6 s m$ & Ningxia \\
\hline 4 & Zhongwei & $54.69 \%$ & 2.79 & $2 \mathrm{~B}$ & $2 n=20=14 m+6 s m$ & Ningxia \\
\hline 5 & Suoyuan & $55.41 \%$ & 2.45 & $1 \mathrm{~B}$ & $2 n=20=14 m+6 s m$ & Shanxi \\
\hline 6 & Changtu & $54.69 \%$ & 1.86 & $2 \mathrm{~A}$ & $2 n=20=14 m+6 s m$ & Liaoning, Inner Mongolia \\
\hline 7 & Xiangyun & $57.97 \%$ & 2.32 & $1 \mathrm{~B}$ & $2 n=20=18 \mathrm{~m}+2 \mathrm{sm}$ & Yunnan, Sichuan \\
\hline 8 & Changyuan & $57.90 \%$ & 2.66 & $1 \mathrm{~B}$ & $2 n=20=18 \mathrm{~m}+2 \mathrm{sm}$ & Yunnan \\
\hline 9 & Wudu & $56.11 \%$ & 2.99 & $2 \mathrm{~B}$ & $2 n=20=14 \mathrm{~m}+6 \mathrm{sm}$ & Gansu, Ningxia \\
\hline 10 & Pingyu & $56.75 \%$ & 3.06 & $2 \mathrm{~B}$ & $2 n=20=14 \mathrm{~m}+6 \mathrm{sm}$ & Shandong \\
\hline 11 & Yancheng & $59.25 \%$ & 1.91 & $1 \mathrm{~B}$ & $2 n=20=18 \mathrm{~m}+2 \mathrm{sm}$ & Shandong \\
\hline 12 & Xiangyang & $54.52 \%$ & 2.03 & $1 \mathrm{~B}$ & $2 n=20=14 m+6 s m$ & Henan \\
\hline 13 & Tongxiang & $56.32 \%$ & 2.05 & $1 \mathrm{~B}$ & $2 n=20=20 \mathrm{~m}$ & Zhejiang, Fujiang \\
\hline 14 & Pinghu & $58.12 \%$ & 2.31 & $2 \mathrm{~B}$ & $2 n=20=18 m+2 s m$ & Zhejiang \\
\hline 15 & Baoji & $56.20 \%$ & 1.79 & $2 \mathrm{~A}$ & $2 n=20=14 \mathrm{~m}+6 \mathrm{sm}$ & Shananxi \\
\hline 16 & Huaiyin & $56.64 \%$ & 2.93 & $1 \mathrm{~B}$ & $2 n=20=20 \mathrm{~m}$ & Jiangsu \\
\hline 17 & Chongqing & $60.27 \%$ & 3.47 & $2 \mathrm{~B}$ & $2 n=20=18 m+2 s m$ & Chongqing \\
\hline 18 & Wenzhou & $56.75 \%$ & 2.34 & $1 \mathrm{~B}$ & $2 n=20=18 \mathrm{~m}+2 \mathrm{sm}$ & Zhejiang \\
\hline 19 & Huangzhong & $61.11 \%$ & 2.69 & $1 \mathrm{~B}$ & $2 n=20=14 m+6 s m$ & Qianghai \\
\hline 20 & Xining & $59.54 \%$ & 1.69 & $2 \mathrm{~A}$ & $2 n=20=12 \mathrm{~m}+8 \mathrm{sm}$ & Qianghai \\
\hline 21 & Changbaishan & $56.13 \%$ & 2.65 & $1 \mathrm{~B}$ & $2 n=20=14 \mathrm{~m}+6 \mathrm{sm}$ & Jilin, Inner Mongolia \\
\hline 22 & Hanma & $59.45 \%$ & 2.83 & $2 \mathrm{~B}$ & $2 n=20=18 \mathrm{~m}+2 \mathrm{sm}$ & Anhui \\
\hline 23 & Yeji & $56.11 \%$ & 3.06 & $2 \mathrm{~B}$ & $2 n=20=14 \mathrm{~m}+6 \mathrm{sm}$ & Anhui, Jiangsu \\
\hline 24 & Xingtai & $56.23 \%$ & 2.03 & $1 \mathrm{~B}$ & $2 n=20=20 \mathrm{~m}$ & Hebei \\
\hline 25 & Xuanhua & $58.14 \%$ & 3.47 & $2 \mathrm{~B}$ & $2 n=20=14 \mathrm{~m}+6 \mathrm{sm}$ & Hebei, Tianjini \\
\hline 26 & Tuoketuo & $55.25 \%$ & 2.23 & $1 \mathrm{~B}$ & $2 n=20=14 \mathrm{~m}+6 \mathrm{sm}$ & Inner Mongolia \\
\hline 27 & Huocheng & $57.23 \%$ & 2.45 & $1 \mathrm{~B}$ & $2 n=20=16 \mathrm{~m}+4 \mathrm{sm}$ & Xinjiang \\
\hline
\end{tabular}




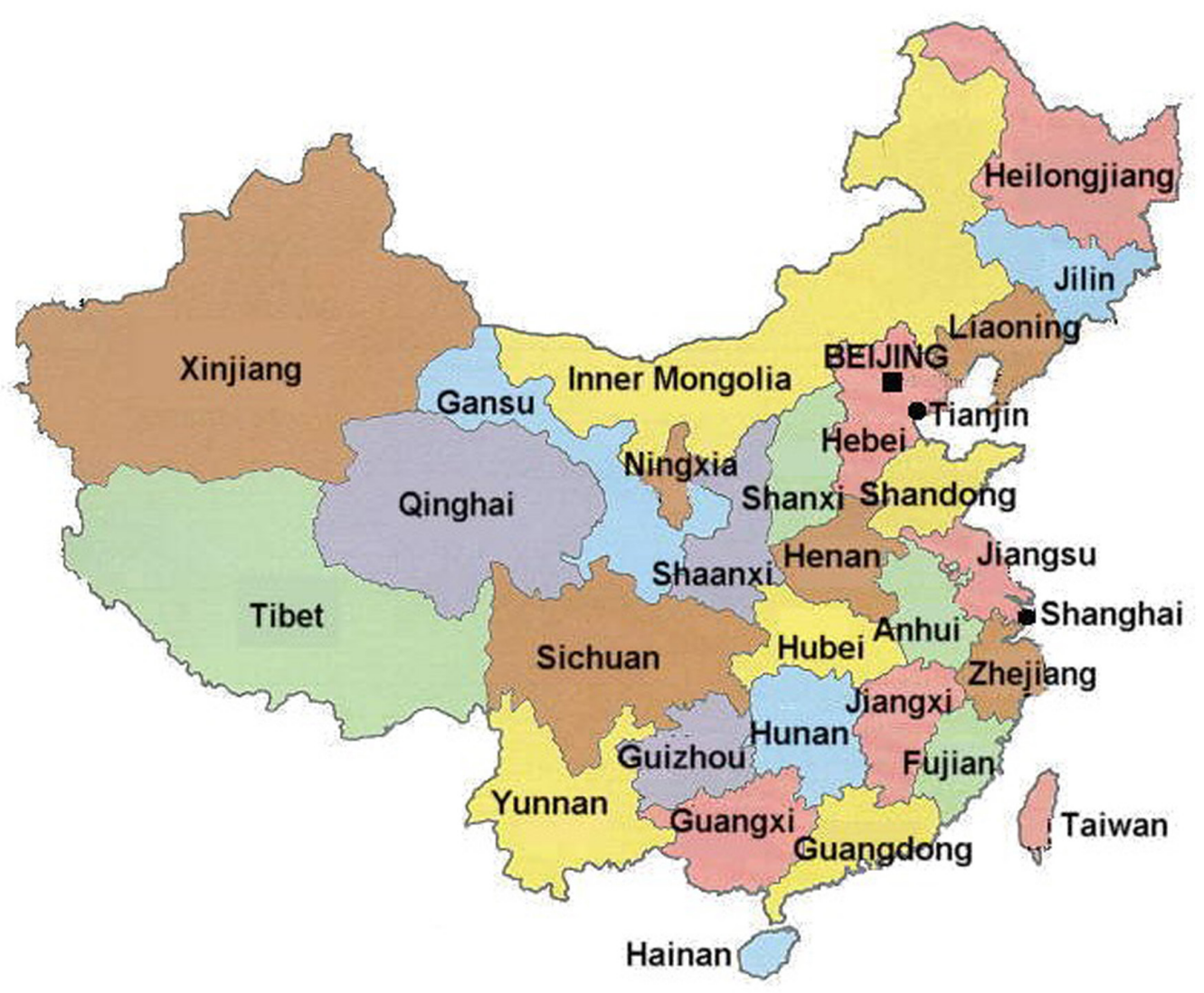

Figure 2. Geographical distribution of provinces in China associated with the distribution of the 27 samples listed in Table 2.

\section{ISSR analysis}

Overall, the 23 ISSR primers amplified 183 bands, of which 157 were polymorphic. Each primer yielded 3-14 bands, with an average of 8.0 bands and 6.8 polymorphic bands per primer (Figure 3 ). The percentage of polymorphic loci was $85.8 \%$, which suggested that the ISSR markers were suitable for the analysis of genetic variation. The high percentage of polymorphic loci was probably caused by differences in the local ecological environment; the 27 native cannabis varieties are widely distributed throughout China. In addition, cannabis is a cross-pollinating crop, and therefore has high heterogeneity.

The highest number of polymorphic loci was found in the native cultivar 'Yeji'; in this cultivar, 83 loci were amplified by the 23 primers and the proportion of polymorphic loci was $52.87 \%$. The native cultivar 'Suoyuan' had the lowest number of polymorphic loci; in this cultivar, 49 loci were amplified by the 23 primers and the proportion of polymorphic loci was only $31.21 \%$. 


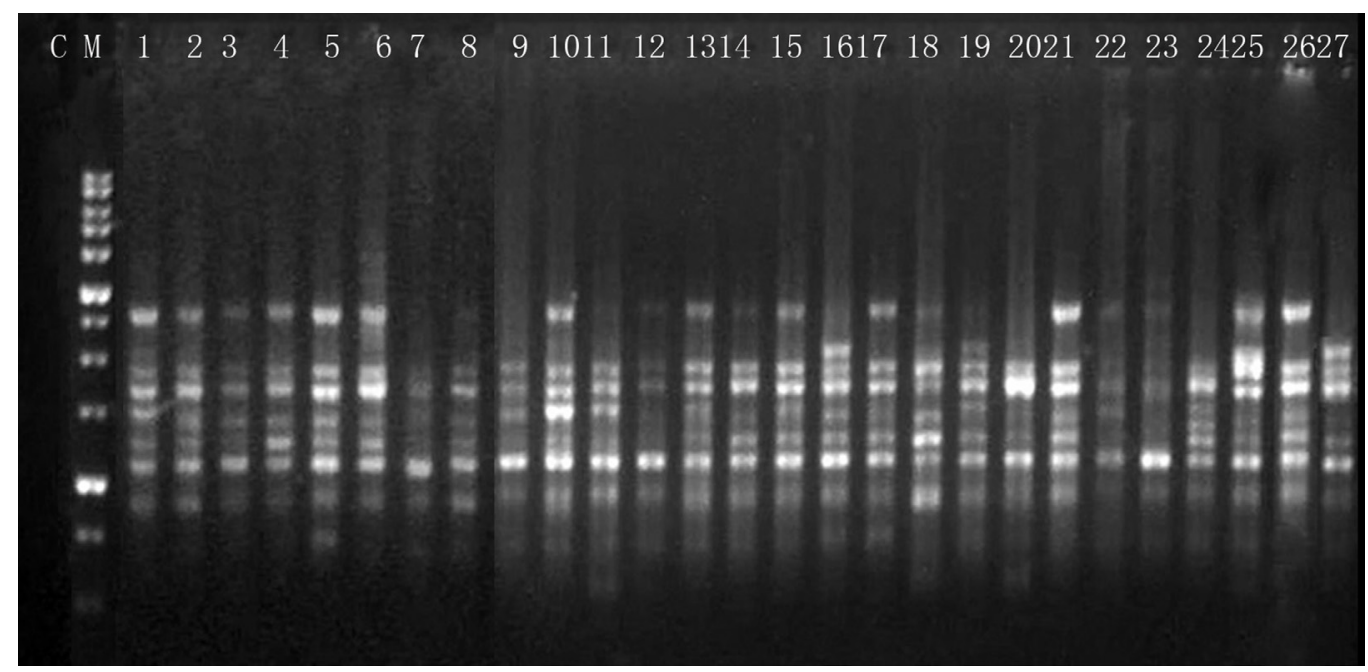

Figure 3. ISSR amplification results of the 27 cannabis cultivars amplified with primer U848 (lanes 1-27 are the same as in Table 2). Lane $M=$ DNA markers (from bottom to top, the bands represent 300, 400, 500, 600, 700, 800, $900,1000,1500,2000,2500$, and $3000 \mathrm{bp})$.

\section{Cluster analysis}

The genetic distances between the 27 cultivars ranged from 0.0511 to 0.5912 (average 0.3297), which implied considerable genetic variation (Table 3 ).

The genetic relationships between the different cultivars were clearly visualized by using the UPGMA based on the genetic distances (Figure 4). At a genetic distance of 0.233 , the 27 native cannabis cultivars were divided into 13 categories. The genetic distance within each category was low; therefore, the native cultivars can be regarded as similar cultivars, which are unsuitable for cross-breeding work. At a genetic distance of 0.324 , the 27 native hemp varieties were divided into 5 categories. At a genetic distance of 0.336 , the 27 native cannabis cultivars were divided into the following 3 categories: type A, including 10 native cultivars; type B, including only 2 native cultivars; and type $\mathrm{C}$, including 15 native cultivars. The largest genetic distance of 0.5912 occurred between 'Xining' (20) and 'Boli' (2), indicating a distant genetic relationship between these 2 cultivars. The second largest genetic distance of 0.5624 occurred between 'Huocheng' (27) and 'Huangcheng' (19). The smallest genetic distance of 0.0511 occurred between 'Boli' and 'Changtu', indicating a close genetic relationship between these 2 cultivars. The genetic distance between 'Tuoketuo' and 'Huangzhong' was also small, namely, 0.0793 .

\section{DISCUSSION}

\section{Analysis of genetic diversity and evolution based on chromosome markers}

In the present study, we have shown that the chromosomes of 27 Chinese native cannabis cultivars consisted of 5 types, including 3 karyotypes, namely, 1B, 2B, and 2A. 


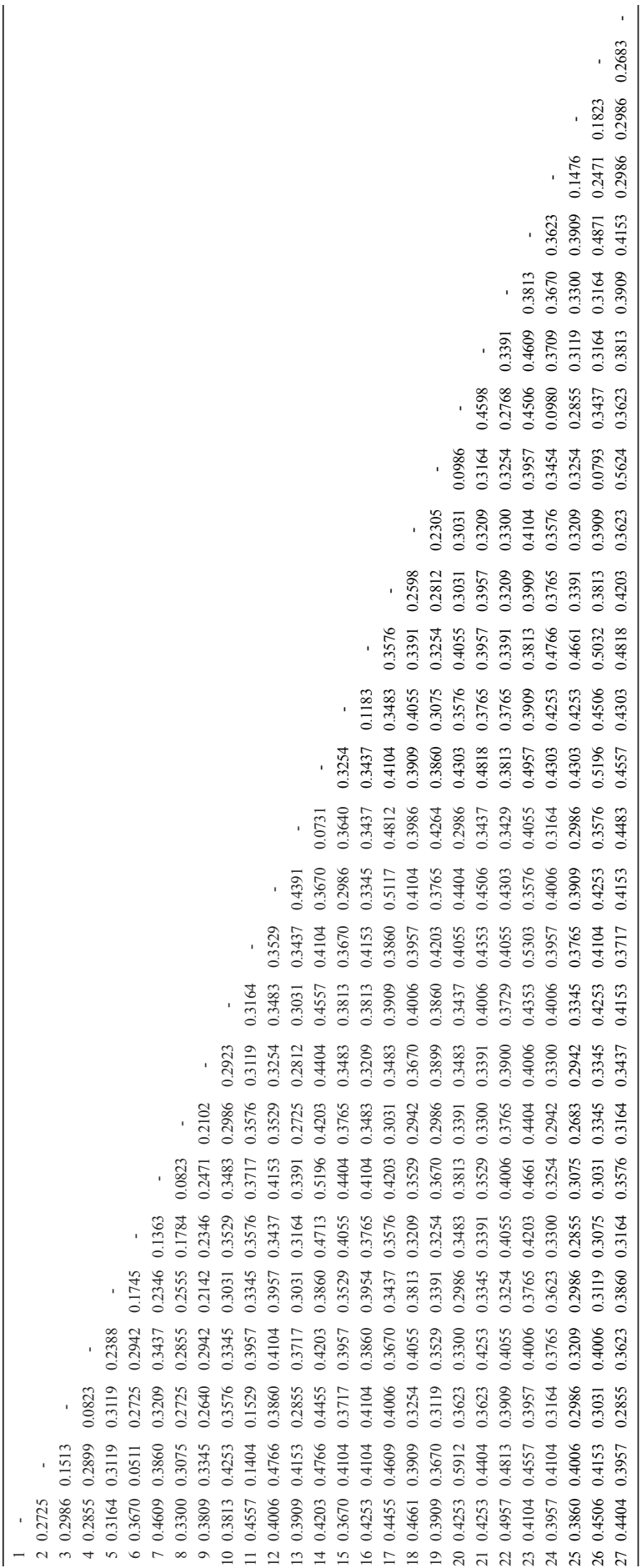


In previous studies, only the karyotypes $1 \mathrm{~B}$ and $2 \mathrm{~A}$ were found, because of a lack of available materials (Guo et al., 1999; Xin et al., 2008). Stebbins (1971) classified karyotypes as $1 \mathrm{~A}$ to $4 \mathrm{C}$, from symmetric to asymmetric; the author further proposed that increasing asymmetry is a tendency of plant evolution. According to this view, all of the native cultivars investigated in the present study belong to relatively primitive types. On the basis of the chromosome length ratio, karyotype, and asymmetry coefficient, 'Wuchang' is a relatively evolved type, whereas 'Changtu' and 'Baoji' represent relatively primitive types. In addition, if a species originated in a particular area, the species should include all types, including relatively primitive types and relatively evolved types. Cannabis is believed to have originated in China. According to the results of our present study, we propose that cannabis originated in southern China and thereafter spread out to north, because the northern germplasm showed relatively evolved types, with enhanced ability to adapt to the environment.

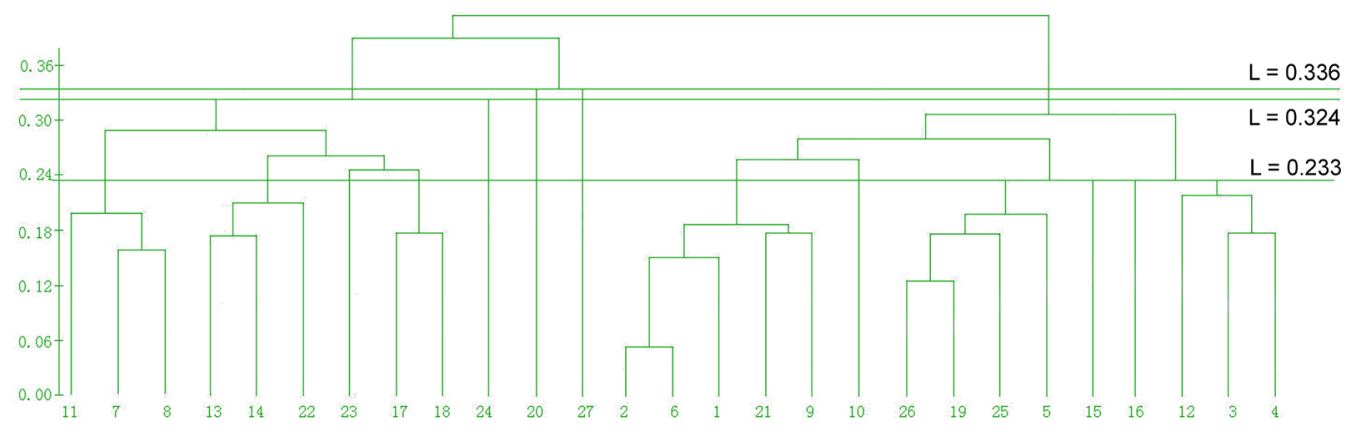

Figure 4. UPGMA dendrogram for the 27 cannabis cultivars, based on ISSR marker polymorphism.

\section{Cluster analysis of genetic diversity based on ISSR markers}

In our ISSR analysis, 183 bands were amplified; 157 of these bands were polymorphic and could be used as a basis for germplasm identification. At a genetic distance of 0.324 , the 27 native hemp varieties were divided into 5 categories that corresponded well with the 5 chromosome constitution types identified by our cytological analysis; these results comprehensively demonstrated interspecific differentiation. Comparison of our present results with morphological data obtained in our previous study (data not shown) reveals certain similarities among different categories, such as central leaflet width and length, main stem color, groove depth on the main stem, and anthocyanin coloration of the crown. However, it is difficult to distinguish germplasm based on morphology alone. Therefore, our previous morphological data partially confirm the accuracy of our present results; however, we have also demonstrated the limitations of using morphological data alone. On the other hand, the genetic relationships between the different cultivars were clearly visualized by using the UPGMA based on the genetic distances between the 27 cultivars.

The proportion of polymorphic loci reflects the capability of a species to adapt to its environment, and thus the potential for continued evolution under conditions of environmental change (Solbrig, 1991). The evolutionary potential of a species and its ability to withstand adverse environmental conditions, as well as the resilience and stability of its ecosystem, are de- 
pendent on genetic diversity (O'Hanlon et al., 2000). Therefore, populations with a higher proportion of polymorphic loci, such as 'Yeji', 'Xining', 'Huocheng', and 'Hanma' have a stronger ability to adapt to environmental change. On the other hand, the native cultivar 'Suoyuan' may disappear in the long term, because of its lower proportion of polymorphic loci.

\section{Analysis of the genetic distribution of cannabis, by using chromosome and ISSR markers}

The genetic distance between varieties is a function of geography. The overall distribution of native cultivars in China shows a geographical pattern; the northern varieties differ from the southern varieties to a certain extent; the small amount of regional hybridity may be the result of adaptation to the local environment, through germplasm exchange. The results of our chromosome and ISSR marker analysis were very similar, indicating that both methods are suitable for the analysis of genetic diversity in cannabis. For a few native cultivars such as 'Tongxiang' and 'Yeji', the results were inconsistent, possibly because chromosome analysis is less responsive to the evolution of variation in the active fragment, whereas chromosome data remain relatively stable within a species. Chromosome markers demonstrate the results of accumulated changes and different stages, whereas ISSR markers enable the quantification of genetic variation.

\section{ACKNOWLEDGMENTS}

We are grateful to Nan Zheng and Yuyan Fang for assistance with germplasm collection and field observation. We thank Professor Kuan Zhang for help with data analysis. Research supported by the Nature Science Foundation of Heilongjiang Province (grant \#QC2012C115) and the Innovation Program of Harbin (grant \#2013RFQYJ014).

\section{REFERENCES}

Babiker HA, Creasey AM, Bayoumi RA, Walliker D, et al. (1991). Genetic diversity of Plasmodium falciparum in a village in eastern Sudan. 2. Drug resistance, molecular karyotypes and the mdr1 genotype of recent isolates. Trans. R. Soc. Trop. Med. Hyg. 85: 578-583.

Bornet B and Branchard M (2001). Nonanchored inter simple sequence repeat (ISSR) markers: reproducible and specific tools for genome fingerprinting. Plant Mol. Biol. Rep. 19: 209-215.

Chen DX, Li LY, Zhang X, Wang Y, et al. (2013). Genetic diversity in wild Dipsacus chinensis populations from China based on ISSR markers. Genet. Mol. Res. 12: 1205-1213.

Culley TM and Wolfe AD (2001). Population genetic structure of the cleistogamous plant species Viola pubescens Aiton (Violaceae), as indicated by allozyme and ISSR molecular markers. Heredity 86: 545-556.

Faeti V, Mandolino G and Ranalli P (1996). Genetic diversity of Cannabis sativa germplasm based on RAPD markers. Plant Breed. 115: 367-370.

Gajera BB, Kumar N and Singh AS (2010). Assessment of genetic diversity in castor (Ricinus communis L.) using RAPD and ISSR markers. Ind. Crops Prod. 32: 491-498.

Guo YL, Xiong HP, Tang SW and Yu CM (1999). Karyotype analysis of hemp (Cannabis sativa L.). China's Fiber Crops 21: $21-23$.

Hong S and Clarke RC (1996). Taxonomic studies of Cannabis in China. J. Int. Hemp. Assoc. 3: 55-60.

Lin L and Hu ZY (2013). Genetic diversity of Camellia japonica (Theaceae), a species endangered to East Asia, detected by inter-simple sequence repeat (ISSR). Biochem. Syst. Ecol. 50: 199-206.

Lin MX and Chen RY (1983). A suggestion on the standardization of karyotype analysis in plants. Wuhan Zhi Wu Xue Yan Jiu 3: 297-302. 
Nagaoka T and Ogihara Y (1997). Applicability of inter-simple sequence repeat polymorphisms in wheat for use as DNA markers in comparison to RFLP and RAPD markers. Theor. Appl. Genet. 94: 597-602.

Nei M and Li WH (1979). Mathematical model for studying genetic variation in terms of restriction endonucleases. Proc. Natl. Acad. Sci. U. S. A. 76: 5269-5273.

O'Hanlon PC, Peakall R and Briese DT (2000). A review of new PCR-based genetic markers and their utility to weed ecology. Weed Res. 40: 239-254.

Passamonti M, Mantovani B and Scali V (1999). Original articles - Animal Diversity and Evolution - Karyotype and allozyme characterization of the Iberian Leptynia attenuata species complex (Insecta Phasmatodea). Zool. Sci. 169: 675-684.

Qian W, Ge S and Hong DY (2001). Genetic variation within and among populations of a wild rice Oryza granulata from China detected by RAPD and ISSR markers. Theor. Appl. Genet. 102: 440-449.

Rubio-Moraga A, Candel-Perez D and Lucas-Borja ME (2012). Genetic diversity of Pinus nigra Arn. populations in southern Spain and northern Morocco revealed by inter-simple sequence repeat profiles. Int. J. Mol. Sci. 13: 5645-5658.

Solbrig OT (1991). From genes to ecosystems: A Research Agenda for Biodiversity. Report of a IUBS-SCOPE-UNESCO Workshop, Harvard Forest, Petersham.

Stebbins GL (1971). Chromosomal Evolution in Higher Plants. Edward Arnold, London.

van Bakel H, Stout JM, Cote AG, Tallon CM, et al. (2011). The draft genome and transcriptome of Cannabis sativa. Genome Biol. 12: R102.

Vicente MJ, Segura F and Aguado M (2011). Genetic diversity of Astragalus nitidiflorus, a critically endangered endemic of SE Spain, and implications for its conservation. Biochem. Syst. Ecol. 39: 175-182.

Wang HF, Zong XX, Guan JP, Yang T, et al. (2012). Genetic diversity and relationship of global faba bean (Vicia faba L.) germplasm revealed by ISSR markers. Theor. Appl. Genet. 124: 789-797.

Wibowo AC, Mohanty AK and Misra M (2004). Chopped industrial hemp fiber reinforced cellulosic plastic biocomposites: thermomechanical and morphological properties. Ind. Eng. Chem. Res. 43: 4883-4888.

Williams JG, Kubelik AR, Livak KJ, Rafalski JA, et al. (1990). DNA polymorphisms amplified by arbitrary primers are useful as genetic markers. Nucleic Acids Res. 18: 6531-6535.

Wu CJ, Cheng ZQ and Huang XQ (2004). Genetic diversity among and within populations of Oryza granulata from Yunnan of China revealed by RAPD and ISSR markers: implications for conservation of the endangered species. Plant Sci. 167: 35-42.

Xin P, Yang M and Shang X (2008). Chromosome number and karyotype analysis of somatic cells of Cannabis sativa. J. West China For. Sci. 37: 66-68.

Zhang L, Zhang HG and Li XF (2013). Analysis of genetic diversity in Larix gmelinii (Pinaceae) with RAPD and ISSR markers. Genet. Mol. Res. 12: 196-207.

Zietkiewicz E, Rafalski A and Labuda D (1994). Genome fingerprinting by simple sequence repeat (SSR)-anchored polymerase chain reaction amplification. Genomics 20: 176-183. 